\title{
Valoración de la docencia de pregrado de urología por docentes y alumnos en el nuevo escenario hospitalario (autogestionado en red)*
}

\author{
JUSTO BOGADO S. ${ }^{\text {a }}$, MARIANA BOGADO C. ${ }^{3 b}$, \\ ILSE LÓPEZ C. ${ }^{2}$, EDUARDO ROSSELOT J. ${ }^{2}$
}

\section{The effects of a new model of hospital management on undergraduate teaching of urology}

Background: Since January 2005, a new model for hospital coordinated assistance was implanted in Chile, denominated "Self Managed Hospitals in net", to improve resource use effectiveness and efficiency. This new design changed health care and teaching models. Aim: To analyze, understand and to reflect on how teachers and students of the Urology Unit of the Eastern Campus of the Faculty of Medicine in the University of Chile, perceive learning in this new hospital scenario. Material and Methods: A qualitative methodology was used, including semi-structured interviews to chief teachers and focal groups of teachers and students. Also, a written structured questionnaire was answered by a group of $5^{\text {th }}$ year students and interns. Results: University teachers perceive that undergraduate learning is affected in the new hospital scenario. Students think that they have less opportunities to directly interact with patients, and therefore have fewer possibilities to take medical histories, perform physical examinations, and fewer occasions to discuss cases with their tutors. Conclusions: The new health system that runs hospitals under a network could jeopardize undergraduate teaching. This is the case for the Urology Service at Hospital and the corresponding Department of Specialties, where the dominant perception of teachers and a number of students is that their clinical learning is endangered by these innovations. To obtain the learning objectives of the undergraduate program in this subject, reorientation of their ambulatory practice and derivation skills must be rationally elaborated to improve student's accomplishment.

(Rev Med Chile 2010; 138: 437-443).

Key words: Education, medical, undergraduate; Financial management, hospital; Qualitative research.

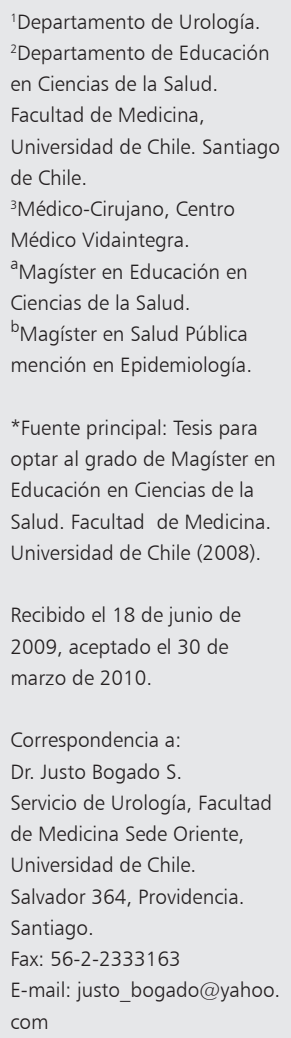

L os nuevos hospitales autogestionados en red instalados en Chile desde enero de 2005 Unstituciones conectivamente integradas en red digital, con desarrollo previsto en todo ámbito: recursos humanos, gestión, satisfacción del usuario, estabilidad financiera y presupuesto autónomo ${ }^{1}$. El propósito al instalar este sistema ha sido elevar la eficiencia y eficacia de los recursos disponibles.

El nuevo diseño ha cambiado el proceso de atención médico-quirúrgico y los procesos clínicos de aprendizaje, $y$ desde esta perspectiva han aparecido claramente dos tipos de pacientes: el paciente de urgencia para ser estudiado de inmediato, y el 
paciente programado. Este último, que es el que mayoritariamente influye hoy en la formación de los estudiantes de pregrado, ya no permanece varios días hospitalizado para operarse, como era antes del año 2005, debido a que las acciones médicas preoperatorias son realizadas en el policlínico o en el consultorio primario de asistencia ${ }^{2}$. Este paciente llega estudiado, documentado y en condiciones de ser intervenido quirúrgicamente, sin previa hospitalización, lo que además es una manera de contener costos ${ }^{2}$. Esta situación induce la necesidad de modificar la docencia, especialmente para los alumnos de $5^{\circ}$ año, debido a que ellos ya no cuentan, en el área de hospitalizados, con los pacientes adecuados en quienes realizar anamnesis, examen clínico y, menos aún, tener oportunidad para discutir casos clínicos; todo lo cual es relevante para el aprendizaje de la especialidad, y la capacitación del futuro profesional (Bogado J. Rediseño de la Práctica Docente en Urología de Pregrado, en el Nuevo Sistema de Salud Predominantemente Ambulatorio. Rosselot E. (dir). Tesis de Magíster. Universidad de Chile, Facultad de Medicina, Departamento de Educación en Ciencias de la Salud. Chile, 2008 [Disponible en Biblioteca de la Sociedad de Educación en Ciencias de la Salud]).

El sistema de salud público ha sido un lugar privilegiado para el aprendizaje clínico y el logro de los objetivos planteados en la formación de los estudiantes; especialmente, cuando, como en nuestro país, las actividades que realizan son expresión de las prioridades en las políticas de salud y, en razón de ello, de las competencias indispensables de sus profesionales. Es responsabilidad de los docentes buscar el adecuado ajuste entre los objetivos asistenciales, aunque sean contingentes, y los de formación profesional y técnica que les compete.

El objetivo principal de este estudio ha sido analizar, comprender y reflexionar en torno a las percepciones de los docentes y estudiantes de la Unidad de Urología del Hospital del Salvador, Campus Oriente, Facultad de Medicina, Universidad de Chile, respecto a las consecuencias que el cambio del modelo asistencial ha producido en la docencia. Del consiguiente debate debiera derivarse una acción remedial que resguarde al sistema de las falencias ya detectadas, y otras de presumible aparición, sin retrotraer la asistencia a un proceso que reduzca la atención ambulatoria. Cualquiera sea la intervención preventiva, esa actividad debe mantenerse como el principal y prioritario cauce de la asistencia en salud, y la docencia tiene que ser hecha con calidad y competencia, en ese ámbito ${ }^{3}$.

\section{Material y Método}

Este estudio se realizó en el Servicio de Urología del Hospital del Salvador, el cual posee un convenio Docente-Asistencial con la Facultad de Medicina de la Universidad de Chile, la cual imparte la asignatura de pregrado de Urología según lo estipulado en el programa de formación general de Medicina mediante un Curso realizado en $5^{\circ}$ año y un Internado de la especialidad en $6^{\circ}$ año.

La metodología de esta investigación se ha inscrito dentro del paradigma cualitativo, en base al estudio de casos, en el cual "el análisis de los datos se centran en un fenómeno seleccionado por el investigador para entenderlo cabalmente, independiente del número de escenarios o de participantes en el estudio" 4 .

Para obtener percepción de cómo se valora la docencia de pre-grado de Urología, se definieron informantes claves entre docentes y alumnos. A todos ellos se aplicó diversos instrumentos propios de la metodología cualitativa ${ }^{5}$ :

a) Entrevistas semi estructuradas a docentes de la especialidad de Urología, incluyendo el Director del Departamento respectivo de la Facultad, Profesores de la más alta jerarquía académica y un Profesor Titular de una universidad regional; b) Grupos focales de 17 estudiantes de $5^{\circ}$ año en 2007, y 10 docentes de Urología; c) Cuestionario estructurado a 12 estudiantes de $5^{\circ}$ año, que cursaron la asignatura con el nuevo sistema de atención hospitalario y a 17 internos de $7^{\circ}$ año, que la cursaron con el antiguo sistema, el año 2007.

Siguiendo el criterio de Kitzinger ${ }^{6}$ se establecieron grupos homogéneos, que facilitaran el análisis en profundidad de aquellos temas comunes a los miembros del grupo. Las preguntas utilizadas para los docentes universitarios se resumen en la Tabla 1 y las usadas en el grupo focal de docentes y alumnos, en la Tabla 2.

Los instrumentos utilizados para obtener la información procurada a través de las preguntas precedentes, fueron validados por tres expertos en metodología cualitativa y docencia universitaria (Bogado J. Rediseño de la Práctica Docente en Urología de Pregrado, en el Nuevo Sistema de Salud Predominantemente Ambulatorio. Rosselot E. (dir). Tesis de Magíster. Universidad de Chile, Fa- 
Tabla 1. Preguntas utilizadas para los docentes universitarios

1. ¿Cómo se percibe la docencia de pre-grado de Urología en el nuevo escenario que ofrece el hospital?

2. ¿Qué ventajas y desventajas ofrece el nuevo hospital autogestionado en red, para la docencia de Urología de pre-grado?

3. Actualmente se percibe un divorcio entre el hospital y la universidad, ¿Qué opina usted?

4. Hay quienes piensan que el hospital privilegia al postgrado, por obtener de él mano de obra barata, en desmedro del pre-grado que está desprotegido, ¿Qué opina usted al respecto?

5. Si Ud. percibe algún tipo de dificultades en la enseñanza de la Urología actual ¿Cómo cree que debe solucionarse? ¿Qué estrategia utilizaría?

6. Actualmente los alumnos disponen de 2 horas para atención en policlínico, durante 8 a 10 días, con reducción paralela de la práctica hospitalaria ¿Cuál es su opinión al respecto?

7. En algunos países la enseñanza de pre-grado de Urología ha disminuido, con tendencia quizás a desaparecer ¿Qué opina Ud. sobre ello?
Tabla 2. Preguntas utilizadas en el grupo focal de docentes y alumnos

1. ¿Cuál cree Ud. que sea el nivel de cumplimiento de las competencias de la asignatura de Urología en el escenario del Hospital Autogestionado? (se refiere a pacientes que llegan de su casa al pabellón; son atendidos por diferentes médicos en cada etapa y son distribuidos en diferentes salas, etc)

2. ¿Cuáles ventajas ofrece el nuevo hospital para realizar la docencia?

3. ¿Cuáles son las desventajas que tiene el nuevo hospital para realizar la docencia?

4. ¿Qué estrategias de enseñanzaaprendizaje propondría(n) usted(es) para superar las dificultades que plantea el nuevo sistema?

5. ¿Desearía(n) agregar algo más?

Tabla 3. Dimensiones y categorías obtenidas a través de las entrevistas y grupos focales dentro las cuales se anotan algunas expresiones textuales, representativas

\begin{tabular}{|c|c|c|c|c|c|}
\hline \multirow{2}{*}{$\begin{array}{l}\text { Actores } \\
\text { consultados }\end{array}$} & \multicolumn{5}{|c|}{ Dimensiones determinadas } \\
\hline & $\begin{array}{c}\text { Ventajas del } \\
\text { nuevo sistema }\end{array}$ & $\begin{array}{l}\text { Desventajas } \\
\text { del nuevo } \\
\text { sistema }\end{array}$ & $\begin{array}{l}\text { Valoración de } \\
\text { la docencia } \\
\text { de pregrado }\end{array}$ & $\begin{array}{c}\text { Valoración del } \\
\text { paciente }\end{array}$ & $\begin{array}{l}\text { Valoración } \\
\text { de la infraes- } \\
\text { tructura del } \\
\text { hospital }\end{array}$ \\
\hline $\begin{array}{l}\text { Docentes universitarios } \\
\text { (individuales) }\end{array}$ & & & & & \\
\hline $\begin{array}{l}\text { Docentes universitarios } \\
\text { (en grupos) }\end{array}$ & & & & & \\
\hline $\begin{array}{l}\text { Estudiantes } \\
\text { (Grupos) }\end{array}$ & & & & & \\
\hline
\end{tabular}

cultad de Medicina, Departamento de Educación en Ciencias de la Salud. Chile, 2008 [Disponible en Biblioteca de la Sociedad de Educación en Ciencias de la Salud]).

Para realizar este estudio se informó a todos los actores involucrados del propósito de la investigación y se obtuvo, también, un consentimiento informado verbal aprobado por los informantes (grabado en formato digital y cassette) y se les aseguró la confidencialidad de los datos y el anonimato. La información obtenida solamente sería utilizada para los fines propios de este estudio y los resultados obtenidos dados a conocer a la comunidad científica. No se advirtió que existieran conflictos de intereses en este estudio.

La información fue recogida y luego reducida a dimensiones y categorías definidas previamente por los investigadores y emanadas de los objetivos de la investigación. Con estos datos se configuró una matriz, cuyo facsímil se expone en la Tabla 3, donde se anotaron las respuestas obtenidas a través de las entrevistas y grupos focales, destacándose 
las dimensiones y categorías obtenidas a través de las entrevistas y grupos focales.

\section{Resultados}

A continuación, transcribimos un consolidado de las respuestas de acuerdo a las dimensiones determinadas instrumentalmente.

\section{Ventajas y Desventajas del Nuevo Sistema}

Sumariamente, los docentes no ven ventajas para la enseñanza, sino más bien perjuicio en ésta. Manifiestan falta de un compromiso de la universidad y del hospital, en que se procuren beneficios compartidos para ambas instituciones y sus usuarios.

Los docentes universitarios en grupo manifiestan que no encuentran ventaja para la docencia en el hospital autogestionado en red. Mientras que los alumnos ven ventajas en la cirugía resolutiva ambulatoria, ya que se asocia a una atención de calidad para el paciente, a pesar de que la relación médico paciente pueda verse deteriorada. Algunas de las expresiones textuales sobre las ventajas y desventajas del nuevo sistema, se exponen en la Tabla 4.

\section{Valoración de la Docencia de Pregrado}

Los docentes insisten en que la enseñanza de pregrado de Urología debiera estar en el hospital, ya que la mayoría de estos pacientes pasan por pabellón. Piensan que hay retroceso de la docencia de pregrado, ya que los alumnos se están alejando del paciente. Enfatizan que se debe buscar otra forma de hacer docencia. Consideran que la gestión hospitalaria ha mejorado en sus números, pero la docencia se ha deteriorado. Los alumnos, por su parte, dicen que sería interesante ver al paciente en evolución, desde el principio del problema hasta su completa resolución. Algunas de las expresiones textuales sobre la valoración de la docencia de pregrado, se exponen en la Tabla 5.

Tabla 4. Expresiones textuales sobre las ventajas y desventajas del nuevo sistema

\begin{tabular}{|c|c|c|}
\hline $\begin{array}{l}\text { Docentes Universitarios } \\
\text { (Individuos) }\end{array}$ & $\begin{array}{l}\text { Docentes Universitarios } \\
\text { (Grupo Focal) }\end{array}$ & Estudiantes \\
\hline $\begin{array}{l}\text { "...todo se ve igual..." } \\
\text { " Falta en el sistema, compromiso } \\
\text { universitario y del hospital para mutuo } \\
\text { beneficio..." } \\
\text { "Si equitativamente se compartieran } \\
\text { docentes, profesionales e infraestruc- } \\
\text { tura física, podría ofrecerse buena } \\
\text { docencia..." } \\
\text { "...Aún tenemos ventajas competitivas } \\
\text { respecto a países más desarrollados... } \\
\text { porque aquí los alumnos tienen con- } \\
\text { tacto directo con el paciente; pero esto } \\
\text { está cambiando últimamente..." } \\
\text { "El nuevo escenario ha creado dificul- } \\
\text { tades, ha afectado el real manejo del } \\
\text { servicio. Nosotros realizamos clases } \\
\text { teóricas y hay poca presencia de los } \\
\text { alumnos; graban las clases" } \\
\text { "... no hacemos reuniones clínicas ni } \\
\text { pasamos visitas". } \\
\text { "Se debe reestructurar el } 5^{\circ} \text { año ya que } \\
\text { en internado se consolidan las cosas" }\end{array}$ & $\begin{array}{l}\text { "...no le encontramos ventaja alguna } \\
\text { para la docencia, la que se ve perju- } \\
\text { dicada" } \\
\text { "...La docencia de pregrado es inade- } \\
\text { cuada; estamos mostrando a los alum- } \\
\text { nos una medicina deshumanizada, que } \\
\text { sin duda va a inferir en lo formativo" } \\
\text { "El daño mayor es que se ha perdido } \\
\text { la secuencia tutorial; no hay consenso } \\
\text { para tener una discusión operatoria, } \\
\text { no se discute. El alumno no ve que } \\
\text { haya un criterio de diagnóstico, ni de } \\
\text { manejo frente al enfermo, y esto es } \\
\text { muy grave" } \\
\text { "...el paciente llega listo desde su casa } \\
\text { para ser operado, y el alumno ve al } \\
\text { paciente con los hechos consumados; } \\
\text { por lo tanto no hay discusión y, desde } \\
\text { ese punto de vista, el pregrado está } \\
\text { perjudicado." } \\
\text { "...docencia inadecuada para el pre- } \\
\text { grado". } \\
\text { "Debe haber una politica de la Univer- } \\
\text { sidad para que las escuelas de medi- } \\
\text { cina tengan buen campo clínico para } \\
\text { realizar la docencia". }\end{array}$ & $\begin{array}{l}\text { "cuando se logre el funcionamiento } \\
\text { con autogestión se dará al paciente } \\
\text { una atención de calidad". } \\
\text { "Lo malo es que los pacientes pasan } \\
\text { por varias manos; tratan la enfermedad } \\
\text { pero no al enfermo, hay poca relación } \\
\text { médico-paciente" }\end{array}$ \\
\hline
\end{tabular}


Valoración de la docencia de pregrado de urología en el nuevo escenario hospitalario - J. Bogado S. et al

\section{Valoración del Paciente}

Los docentes opinan que aún Chile tiene ventajas comparativas a este respecto, frente a países más desarrollados; esto porque los alumnos tienen mayor contacto con los pacientes, pero están igualmente conscientes de que tal situación, últimamente, está cambiando. Los estudiantes creen que existe mala docencia. Los alumnos opinan que ellos son demasiados para ver o examinar un paciente, tanto en el policlínico como en el postoperatorio, y que les falta privacidad. Algunas de las expresiones textuales sobre la valoración del paciente se exponen en la Tabla 6 .

\section{Valoración de la Infraestructura Física del Hospital}

Todos los actores involucrados coinciden en que el hospital carece de infraestructura adecua-

Tabla 5. Expresiones textuales sobre la valoración de la docencia de pregrado

\section{Docentes Universitarios (Individuos)}

"La Urología de pregrado debe ser realizada en el hospital porque la atención primaria no es para especialidades; salvo la infección urinaria, la mayoría pasan por pabellón quirúrgico y eso no se puede concretar en los consultorios externos"

"La docencia de pregrado está yendo para atrás, los alumnos se están alejando del paciente, debemos buscar otras formas de hacer docencia". "(...por ejemplo en forma virtual)"

\section{Docentes Universitarios (Grupo focal)}

"El problema mayor es para los alumnos de $5^{\circ}$ año, y no para los internos que se pegan al docente teniendo un manejo más adecuado de los pacientes; incluso participan en el pabellón quirúrgico"

"...Actualmente la gestión hospitalaria ha mejorado. Mejoraron los números, pero la docencia está francamente deteriorada..."

\section{Estudiantes}

"...La docencia está bien..."

"...Sería interesante ver al paciente en forma progresiva, desde el principio y seguirlo"

"...somos muchos para ver un paciente"

"...Hay poca práctica semiológica..."

Tabla 6. Expresiones textuales sobre la valoración del paciente

\section{Docentes Universitarios (Individuos)}

"...lo que aparentemente, para nosotros son debilidades...se trasforman en fortalezas"

"...aquí los alumnos tienen más contacto directo con los pacientes, pero eso está cambiando últimamente"

"...la autogestión en red no tiene relación con las malas consecuencias para la docencia..."

\section{Docentes Universitarios} (Grupo Focal)

"...Los pacientes son devueltos a sus domicilios ya que no pueden operarse por falta de camas,... hay un desastre funcional y docente"

"...además (de los pacientes) tenemos 7 a 10 estudiantes que se nos asigna a cada docente. Yo pondría un policlínico especial para docencia"

\section{Estudiantes}

"...La visita a los pacientes operados, como somos muchos, es inadecuada..."

"...lo mismo sucede en el policlínico adosado, donde somos muchos para ver un paciente"

"...los pacientes no tienen privacidad..."

Tabla 7. Expresiones textuales sobre la valoración de la infraestructura física del hospital

\section{Docentes Universitarios} (Individuos)

"...el hospital no dispuso una infraestructura física adecuada y la universidad nunca puso un número adecuado de docentes..."

"Se debe tener mucho cuidado, ya que hay universidades deseosas de pagar por docencia...eso es apetitoso para un director de hospital..."

\section{Docentes Universitarios} (Grupo Focal)

"... La escuela de medicina debe tener un campo clínico adecuado para realizar docencia..."

"...La docencia de pregrado tendría que estar fundamentalmente en el policlínico adosado; sin embargo, no estamos preparados para ello ya que la carga asistencial es muy alta..."

\section{Estudiantes}

"La parte física del hospital es inadecuada, los pacientes no tienen privacidad..." 
da. Los docentes opinan que, si tanto el hospital como la universidad contribuyeran a mejorar la estructura física hospitalaria y hubiera un número adecuado de docentes, no habría tantos problemas docente-asistenciales. Los alumnos, además, creen que la Escuela de Medicina debería disponer de campos clínicos más apropiados, especialmente, cuando se atienden pacientes que requieren mayor privacidad en su asistencia y durante su colaboración a los aprendizajes. Algunas de las expresiones textuales sobre este tema, se exponen en la Tabla 7.

\section{Discusión}

Los cambios experimentados en los últimos años por el Sistema de Salud en Chile han determinado que los hospitales deban autogestionarse, lo cual representa un nuevo escenario para la educación en salud. La misión principal de un centro asistencial es proporcionar acciones de salud a la población en forma oportuna, resolutiva y eficiente. Sin embargo, los Centros Docente-Asistenciales, como el Hospital del Salvador, también incluyen en su misión, la formación técnica y profesional (pre y postgrado $)^{7}$, debiendo estar en esto fundidos con la misión de la Facultad de Medicina de la Universidad de Chile ${ }^{8}$. Es necesario conciliar ambas visiones para, de esta forma, obtener una atención de calidad junto a una docencia de excelencia, para la salud de hoy y mañana.

Las modificaciones realizadas al Sistema de Salud han traído como consecuencia que el Hospital del Salvador sea predominantemente ambulatorio $^{7-9}$, lo cual está exigiendo renovar la docencia tradicional de Urología de pregrado, hospitalaria. Esta modalidad afecta especialmente a los alumnos de $5^{\circ}$ año, quienes no tienen ocasión de examinar al paciente en el preoperatorio, lo que previamente hacían, porque éste llega desde la red asistencial generalmente ya programado; es decir, hecho el diagnóstico y la indicación, documentado y listo para ser intervenido quirúrgicamente. Este escenario, desde luego asistencialmente privilegiado, conlleva dificultades para formar en algunas competencias necesarias a un médico general, como son realizar un buen, pertinente y completo interrogatorio y examen clínico, y la discusión procedente a las alternativas terapéuticas, médicas o quirúrgicas. Sin duda, ello requiere con urgencia, que Unidades Académicas y Servicios de Salud en sus áreas convergentes, aúnen esfuerzos para terminar con la disociación docente asistencial reinante y asumir, realmente, el carácter de Centros Docente-Asistenciales Integrados ${ }^{9}$, y lleven a cabo en conjunto, o al menos en coherente asociación, la idónea formación del tan pregonado capital técnico-profesional avanzado que nuestra nación reclama y su salud exige.

Además, y desde un punto de vista ético, es posible que con este modelo de salud, precariamente instalado y no integrado aún, se acentúe el proceso de deshumanización de la relación médico paciente y su insensible contagio a nuestros alumnos; ya que en el afán de maximizar la eficiencia de los recursos materiales, esa comunicación se hace más precaria y fugaz, a medida que diferentes profesionales, sin mayor interacción personal entre ellos, se suceden en su atención ${ }^{10,11}$.

Es así que, los actores relevantes, destacando aspectos sensibles e importantes del nuevo escenario del hospital autogestionado y algunos efectos que inciden en la docencia ${ }^{9-11}$, ratifican que en el corazón del hospital reside lo esencial de la atención de los pacientes. Ésta debe ser siempre de calidad, y respetar los tiempos diagnósticos y de resolución, aspectos que se ven claramente mejorados en el nuevo ámbito clínico. Pero también vemos que esto puede ir en desmedro de la docencia, en el área de la salud. La innovación es imperiosa, por lo tanto, de parte de la Escuela, en el proceso de enseñanza aprendizaje, ante el nuevo modelo hospitalario. También los directivos de los hospitales deben colaborar activamente para evitar el deterioro de dicho proceso, ya que esta docencia está inmersa en la visión y en la responsabilidad del hospital con la sociedad que lo nutre.

Los autores concuerdan, en general, con las opiniones vertidas por docentes y estudiantes en los grupos de discusión, respecto al deterioro de la docencia en el nuevo escenario hospitalario, y en que se debe contextualizar, exponer y analizar las estrategias de innovación docente para responder a los desafíos del nuevo sistema de salud y a las demandas de un proceso de enseñanza aprendizaje de calidad, como lo exige el pregrado. Deberá ponerse énfasis en la profesionalización y desempeño de los docentes con motivación, hacia la innovación que produzca mejoras sustanciales para la práctica clínica, con especial énfasis en las tecnologías de información y comunicación, cen- 
tradas en el aprendizaje, uso de software con casos clínicos interactivos, y pacientes estandarizados, reales o en base a simulaciones.

$\mathrm{Al}$ analizar el resultado de los cuestionarios respondidos por los internos $\left(7^{\circ}\right)$ y por los alumnos $\left(5^{\circ}\right)$, se ven claras diferencias que desfavorecerían a los últimos en cuanto a las competencias logradas, ya que éstos aún no han hecho su residencia y carecen, por lo tanto, de aprendizajes que los primeros ya incorporaron. Este hecho pudiera sesgar los datos; sin embargo, se ha planteado que estas diferencias podrían deberse, también, a que los internos hicieron su pasantía cuando el hospital funcionaba con el sistema antiguo, en que el estudiante tenía mayor contacto con los pacientes que permanecían hospitalizados previo a la cirugía, y no como ocurre actualmente en los hospitales, que son de consulta predominantemente ambulatoria.

No obstante, se considera ciertamente riesgosa la situación actual, ya que el pregrado es justamente para que los estudiantes integren y consoliden en la práctica con los pacientes, antes de especializarse, los conocimientos predominantemente teóricos adquiridos en forma previa. Para que se impregnen de las competencias genéricas no pueden desperdiciar estas instancias de aprendizaje, aunque correspondan a objetivos de la semiología y del examen físico general, aparentemente, pertinentes a otras asignaturas. Por consiguiente, es imperativo disponer de otros escenarios de aprendizaje, que reemplacen a la sala de hospitalización especializada y puedan los alumnos entrenarse ahí para recuperar las capacidades clínicas desfavorecidas con el cambio de modelo asistencial ${ }^{1}$.

Por otro lado, también se concuerda con los estudiantes y directivos hospitalarios en iniciar la docencia de pregrado en la atención ambulatoria, que por lo demás, es considerada en los programas de postítulo atingentes de la Escuela de Medicina de la Universidad de Chile. En este aspecto, la reforma de salud pone énfasis en la atención primaria para resolución de las patologías más frecuentes con implementación de programas de promoción, prevención y rehabilitación.

En definitiva, para la docencia de pregrado, siguiendo el esquema tradicional de aprendizaje, el nuevo sistema de atención plantearía en algunas Unidades Académicas, como el Servicio de Urología del Hospital Del Salvador y el Departamento de Especialidad correspondiente, más desventajas que ventajas, según la impresión predominante de los docentes y de numerosos alumnos. Por todo esto, estamos obligados a formular estrategias de educación consistentes con las diferentes capacidades clínicas que deseamos adquieran en su formación los profesionales; es decir, competencias que incluyan conocimientos, habilidades, destrezas, actitudes, aptitudes y valores esenciales, en correspondencia con los objetivos educacionales propuestos y sus inevitables innovaciones, y en los escenarios adecuados para satisfacer las prioridades sanitarias del país, tanto de hospitalización como de trato ambulatorio ${ }^{2,10}$.

\section{Referencias}

1. Ministerio de Salud (MINSAL). Ley 19.937, Autoridad Sanitaria. Publicada en el Diario Oficial, 24 de febrero, 2004. [Consultado: marzo 11, 2007]. Disponible en: http://www.asrm.cl/sitio/download/normativaseremi/ LEY_19937. PDF.

2. Rosselot E. La atención primaria y el proyecto de reforma de la salud. Bol. Fac. Med. 2003; 3-4: 20.

3. Montoya-Aguilar C, Ipinza M. Un programa para recuperar y mejorar el aporte de los médicos en la atención primaria de salud. Cuad Méd Soc (Chile) 2009, 49: 1625.

4. Taylor S, Bogdan R. Introducción a los Métodos Cualitativos de Investigación. Buenos Aires. Paidos. 1986.

5. Mc Millan J, Schumacher S. Investigación Educativa. $5^{\circ}$ Edición. En: Pearson Addison Wesley; 2005. p. 446.

6. Kitzinger J. Qualitative research. Introducing focus groups. BMJ 1995; 311: 299-302.

7. Hospital del Salvador. Misión y Visión de la Institución. [Consultado: diciembre 29, 2007]. Disponible en: http:// www.hsalvador.cl/Mision.html

8. Universidad de Chile. Misión de la Facultad de Medicina. [Consultado: diciembre 19, 2007]. Disponible en: http://www.med.uchile.cl/presentacion/mision.html

9. Rosselot E. Hacia el médico que nuestros países necesitan: énfasis en la comunicación y en la formación de los docentes. Rev Med Chile 2003; 131: 331-7.

10. Rosselot E. Reivindicando la medicina como profesión científico humanista. Rev Med Chile 2003; 131: 454 -5.

11. Academia Chilena de Medicina. Comité de Educación Superior. Informe sobre la Situación Actual de la Educación Médica en Chile. Conclusiones y Recomendaciones. Serie Monografías Académicas LOM. Ed. Ltda 2008; pp 53-6. 